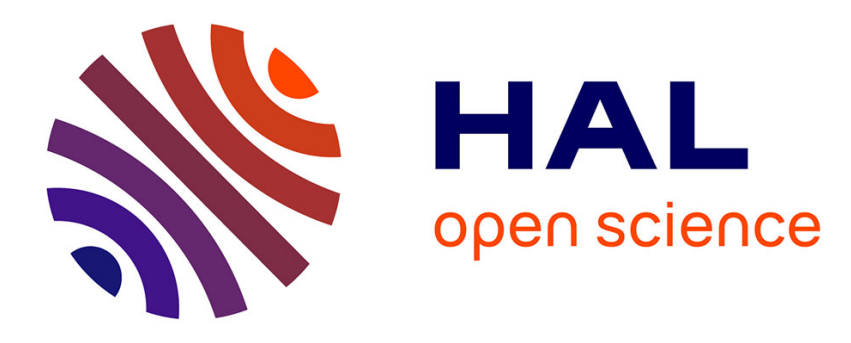

\title{
PERCEPTION DE LA COLORATION D'UN BRUIT BLANC DUE À UN ÉCHO LATÉRAL
}

\author{
P. Boussard, F. Santon
}

\section{To cite this version:}

P. Boussard, F. Santon. PERCEPTION DE LA COLORATION D'UN BRUIT BLANC DUE À UN ÉCHO LATÉRAL. Journal de Physique IV Proceedings, 1992, 02 (C1), pp.C1-221-C1-224. 10.1051/.jp4:1992145 . jpa-00251216

\section{HAL Id: jpa-00251216 https://hal.science/jpa-00251216}

Submitted on 1 Jan 1992

HAL is a multi-disciplinary open access archive for the deposit and dissemination of scientific research documents, whether they are published or not. The documents may come from teaching and research institutions in France or abroad, or from public or private research centers.
L'archive ouverte pluridisciplinaire HAL, est destinée au dépôt et à la diffusion de documents scientifiques de niveau recherche, publiés ou non, émanant des établissements d'enseignement et de recherche français ou étrangers, des laboratoires publics ou privés. 


\title{
PERCEPTION DE LA COLORATION D'UN BRUIT BLANC DUE À UN GCHO LATÉRAL
}

\author{
P. BOUSSARD et F. SANTON \\ Equipe de Psychoacoustique, Environnement Acoustique et Perception, Laboratoire de Mécanique et \\ d'Acoustique du CNRS, 31 chemin Joseph-Aiguier, F-13402 Marseille cedex 09, France
}

\begin{abstract}
In this paper, the coloration, which is a modification of timbre, is more precisely defined. The classical results, concerning coloration of a white noise due to its echo, both with the same frontal direction, are presented. Given the importance of lateral echoes to the quality of room acoustics, we got interested in coloration with a lateral echo. From the first results, it seems that the coloration thresholds are much lower than those with a frontal echo. However, they appear to be the results of two cues: a spectral one (the coloration, we are interested in) and a spatial one. As we reduce step by step the latter one, the results with the lateral echo get closer and closer to the results with a frontal echo. Then, the perception of coloration does not seem to depend on the azimuth of the echo, and our first results were, in fact, based on a spatial cue.
\end{abstract}

\section{1) Précision sur la coloration - Procédure expérimentale}

Dans le domaine de l'acoustique et plus particulièrement de la psychoacoustique, le terme de coloration demande à être précisé. Il désigne, au sens le plus général, une modification de timbre connu (pour un son établi), c'est-à-dire, un changement de spectre perceptible par le système auditif. Dans le domaine de l'acoustique des salles, on dira plus précisément qu'une salle colore, lorsque la modification de timbre (qu'une salle non anéchoïque introduit toujours) a un caractère désagréable, gênant. A l'origine, le terme a certainement été emprunté au domaine de l'optique où, lorsque l'énergie d'une partie du spectre d'une lumière initialement blanche ou grise varie suffisamment, la couleur apparaît. De même en acoustique, la coloration peut désigner l'émergence de parties du spectre qui donne, en plus de la modification de timbre, une sensation de hauteur tonale. C'est le cas de nos expériences.

Nous nous sommes attachés à déterminer la sensibilité à la coloration d'un bruit blanc frontal due à son écho d'azimut variable. L'addition de l'écho retardé et non atténué donne un effet de filtre en peigne sur le bruit blanc. Lorsque l'atténuation de l'écho par rapport au son direct augmente, la forme du filtre en peigne tend vers une sinusoïde dont l'amplitude diminue (l'effet est moins sensible). Pour un azimut et un retard donnés, on détermine l'atténuation de l'écho au seuil de perception de la coloration d'un auditeur placé en chambre anéchoïque où l'on simule le son direct et l'écho. La méthode psychométrique est adaptative de type " 2 down - 1 up" et à choix forcé sur deux intervalles de comparaisons précédés d'un intervalle de référence. Le seuil retenu correspond à une probabilité de $71 \%$ de réponses correctes. 


\section{2) Résultats antérieurs - Orientation des recherches - Premiers résultats}

La sensibilité moyenne au spectre d'un bruit blanc coloré par son écho retardé est connue depuis trente ans [1]. Nous avons retrouvé ces résultats (qui ont été de nombreuses fois confirmés depuis) avec un bruit blanc frontal et son écho de même direction (Fig. 1).

En acoustique des salles, de nombreux travaux [2] [3] ont montré l'importance des réflexions latérales comme facteur de la qualité subjective. Nous avons donc voulu savoir comment était perçue la coloration due à l'écho latéral d'un bruit blanc frontal [4]. Les premiers résultats sont très différents de ceux obtenus avec un écho de même direction que le son direct (Fig. 1). L'allure des courbes ne varie pas dès que l'écho est latéral, que son azimut soit de $15,30,45,60,90$ ou 120 degrés.

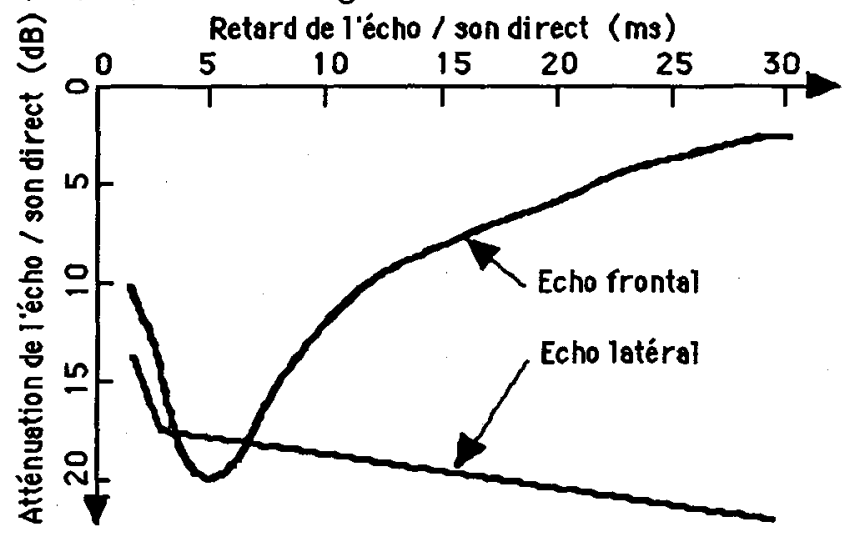

Figure 1: Allure des seuils de coloration pour du bruit blanc

\section{3) Combinaisons des indices spatiaux et de timbre (coloration) - Expériences complémentaires}

Les courbes de la Figure 1 sont très différentes. Nous avons voulu vérifier si cette différence n'était pas due a la présence supplémentaire d'un indice spatial indésirable que nous avions cherché à réduire en utilisant l'effet d'antériorité [5]. En effet, l'auditeur a, en plus de la coloration, une sensation spatiale différente quand il doit comparer le bruit blanc frontal lorsqu'il est seul et lorsqu'il est accompagné par son écho latéral. Cet indice spatial est très perceptible quand l'atténuation de l'écho est faible, cependant la majorité des auditeurs disent se baser sur un indice de timbre (que l'on voudrait être de la coloration) lorsque l'atténuation est plus importante et proche du seuil. Pour évaluer l'influence de cet indice spatial, nous avons mené deux types d'expériences en éliminant d'une part autant que possible les indices spatiaux et en supprimant l'indice de timbre d'autre part.

La suppression de l'indice de timbre (coloration) a été réalisée en gardant les enveloppes de présentation des signaux, mais à l'aide d'un écho non corrélé avec le son direct. Dans ce cas, le test qui se base uniquement sur un indice spatial donne une performance légèrement supérieure à celle obtenue avec l'écho latéral normal. On en déduit que l'indice spatial peut avoir une influence suffisamment importante pour donner le résultat de la Figure 1. Nous n'en sommes néanmoins pas sûrs car il se peut que l'effet d'antériorité sur les signaux eux-mêmes diminue beaucoup l'importance de l'indice spatial mesuré ici.

Deux expériences ont été menées en supprimant les indices spatiaux interauraux (différences d'intensité et de temps d'arrivée entre les oreilles): la première en bouchant l'oreille contralatérale (ce qui n'affecte que très peu la sensibilité à la coloration avec un écho frontal donc à priori avec l'écho latéral ici présent), la deuxième en utilisant un deuxième écho latéral, symétrique du premier par rapport au plan médian. Dans ces deux cas, les seuils obtenus sont quasiment les mêmes; l'allure de la courbe se situe entre celles 
de la Figure 1. Pourtant, dans ces deux expériences complémentaires le signal acoustique arrivant au(x) tympan(s) était coloré de la même façon que dans le cas de l'écho latéral et de l'écoute binaurale. Ceci nous permet de conclure dès maintenant que la courbe obtenue avec l'écho latéral n'est pas due à un indice de timbre mais à un indice spatial.

\section{4) Interprétation et dernière expérience}

On peut alors interpréter la différence que l'on trouve entre le test présentant un écho frontal et ceux utilisant un écho latéral avec élimination des indices spatiaux interauraux. Elle serait due à un indice spatial résiduel. En effet il subsiste dans tous les tests avec écho latéral des indices spatiaux monauraux dâs à la diffraction sur le buste et à la fonction de transfert de l'oreille externe [5]. Nous avons mesuré un gain (Fig 2) qui contient toutes les informations différenciant le stimulus de coloration du test avec l'écho frontal de celui relatif à l'écho latéral.

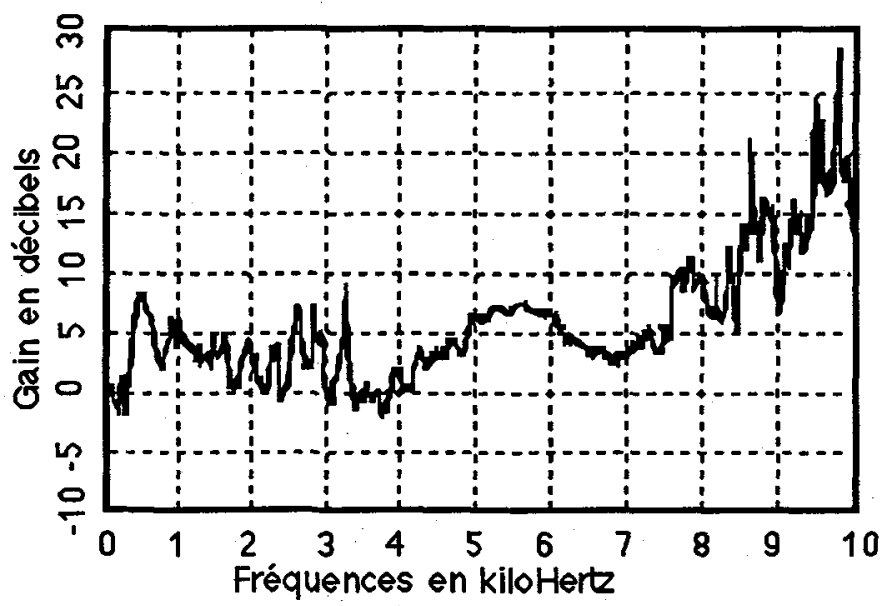

Figure 2: gain d'amplitude en fonction de la fréquence, mesuré au niveau du tympan gauche d'une tête artificielle, du bruit d'une source située à un azimut de 60 degrés à gauche par rapport au bruit de cette même source frontale

Ce gain présente une valeur positive qui globalement augmente avec la fréquence. Il serait donc normal que pour une même atténuation de l'écho, la coloration soit plus sensible avec l'écho latéral. D'autre part, la courbe représentative de ce gain présente de nombreux accidents, résonances et antirésonances aigus. Ceux-ci peuvent être considérés comme un indice spatial monaural.

Pour essayer de diminuer l'influence des indices spatiaux monauraux, nous avons réduit la bande de fréquence à celle strictement utile à la distinction entre le bruit blanc et le bruit blanc coloré par son écho non atténué. Pour un retard supérieur à $5 \mathrm{~ms}$ (ce qui est la zone où se trouvent nos plus grandes différences), il nous a semblé que seule la bande comprise entre $100 \mathrm{~Hz}$ et $2 \mathrm{kHz}$ contenait l'information utile à la perception de la coloration, les fréquences supérieures ayant plutôt un rôle de masquage. D'ailleurs, le nouveau filtrage améliore la perception de la coloration d'environ $3 \mathrm{~dB}$ en condition frontale. La Figure 3 présente les nouveaux seuils de coloration moyens obtenus avec la bande de bruit réduite, l'écho étant situé a 60 degrés sur la gauche de l'auditeur dont l'oreille droite est bouchée.

Cette nouvelle courbe obtenue avec un écho latéral se rapproche encore de nos premiers résultats relatifs à un écho frontal. Nous avons vu que la différence du type d'écoute (monaural/binaural) permettait la comparaison sans correction. Par contre, il est nécessaire de déplacer de $+3 \mathrm{~dB}$ la courbe obtenue avec l'écho frontal, pour tenir compte de l'amélioration apportée par le nouveau filtrage. On peut donc constater que plus on 
supprime des indices spatiaux, plus le seuil de coloration due à un écho latéral est proche de celui obtenu avec un écho frontal. La différence qui subsiste entre la nouvelle courbe et celle que l'on obtiendrait avec un écho frontal et un filtrage identique peut s'expliquer par les indices spatiaux monauraux encore très marqués entre $100 \mathrm{~Hz}$ et $2 \mathrm{kHz}$.

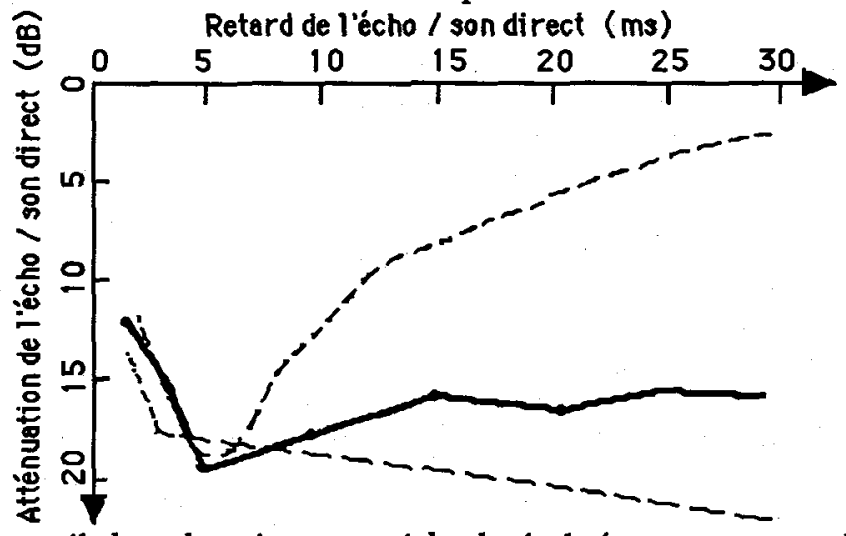

Figure 3: nouveau seuil de coloration avec écho latéral, écoute monaurale du côté de l'écho et bruit à spectre limité (100 $\mathrm{Hz}-2 \mathrm{kHz}$ ). En traits fins pointillés, les résultats de la Figure 1.

\section{5) Conclusion}

Par rapport aux premiers résultats présentés pour la coloration due à un écho latéral, on peut constater que plus on réduit l'importance des indices spatiaux, plus le seuil remonte. Ces seuils bas sont donc dûs à ces indices spatiaux, bien que la majorité des auditeurs se soit basée sur un indice de timbre, selon leurs impressions.

Il est connu que, pour un son pur, une variation d'intensité a pour principal effet psychoacoustique une variation de sonie, et une variation de fréquence, une variation de hauteur. Mais par exemple un son pur d'intensité constante dont la fréquence augmente autour de $2 \mathrm{kHz}$ sera aussi perçu avec une sonie croissante. Ces paramètres physiques peuvent donc agir sur des caractères subjectifs autres que le principal. De même, nous voyons ici qu'une disposition spatiale différente peut modifier le timbre. On sait réciproquement qu'un changement de spectre peut créer une sensation spatiale (réverbération artificielle par exemple) ou même entraîner la localisation d'un son dans une direction différente de celle de sa source.

On peut donc retenir de cette étude qu'à priori la coloration, au sens où on l'entend habituellement comme altération d'un timbre (par exemple celui d'un instrument de musique connu), ne serait guère plus sensible pour du bruit blanc lorsque l'écho qui la provoque est latéral que lorsqu'il est frontal. D'autre part, un écho latéral donne des sensations spatiales et de timbre qui sont très proches et difficilement dissociables par l'auditeur lui-même.

\section{Bibliographie}

[1] B.S. ATAL, M.R. SCHROEDER et K.H. KUTTRUFF, "Perception of coloration in filtered Gaussian noise - Short-time spectral analysis by the ear".Actes du 4eme C.I.A. 1962.

[2] M. BARRON et A.M. MARSHALL, "Spatial impression due to early lateral reflections in concert halls". Journal of sound and vibration. Vol.77, No 2. 1981.

[3] Y. ANDO, "Concert hall acoustics". Springer-Verlag. 1985.

[4] F. SANTON, "coloration d'un bruit blanc par un écho d'incidence latérale". Note de recherche. Acustica. Vol.74, p.94 et 95. 1991.

[5] . J. BLAUERT, "Spatial hearing". The M.I.T. Press. 1983. 